Supporting Information

\title{
Pt/Graphene Catalyst and Tellurium Nanowire-Based Thermochemical Hydrogen (TCH) Sensor Operating at Room Temperature in Wet Air
}

Tae-Yeon Hwang, ${ }^{\dagger}$ Gwang-Myeong Go ${ }^{\ddagger}$ Siwoo Park,,${ }^{\ddagger}$ Jimin Lee, ${ }^{\ddagger}$ YoSeb Song, ${ }^{\S}$ Seil Kim,,$"$ Hong-Baek Cho, ${ }^{\dagger}$ and Yong-Ho Choa ${ }^{*}, \$, \S$

$\dagger$ Center for Quantum Information, Korea Institute of Science and Technology (KIST), Seoul 02792, Republic of Korea

$\$$ Department of Material Science and Chemical Engineering, Hanyang University, Ansan 15588, Republic of Korea

$\S$ Department of Fusion Chemical Engineering, Hanyang University, Ansan 15588, Republic of Korea

" Electrochemistry Department, Korea Institute of Materials Science, Changwon 51508, Republic of Korea

${ }^{*}$ Corresponding author.

Tel.: +82-31-400-5650; Fax: +82-31-400-8128; E-mail address: choa15@hanyang.ac.kr (Y.-H. Choa) 
(a)

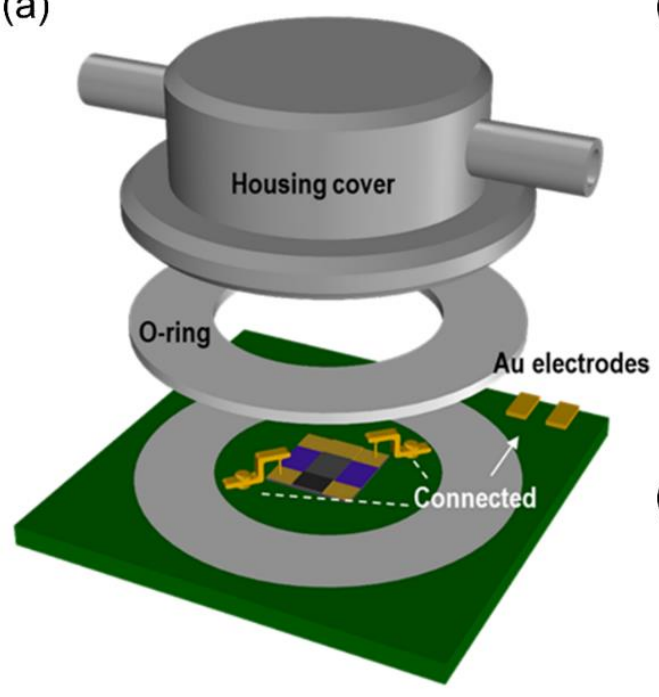

(b)

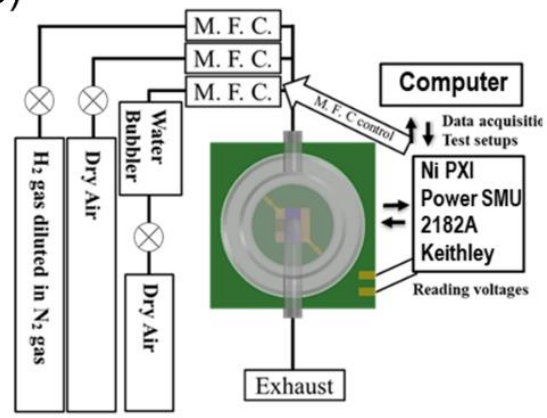

(c)

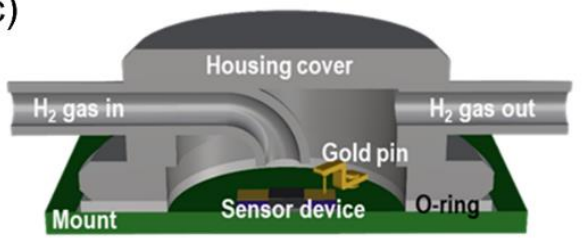

Figure S1. Setups of the gas-sensing system: (a) assembly components, (b) configuration of gas source and device and (c) assembled sensing chamber.
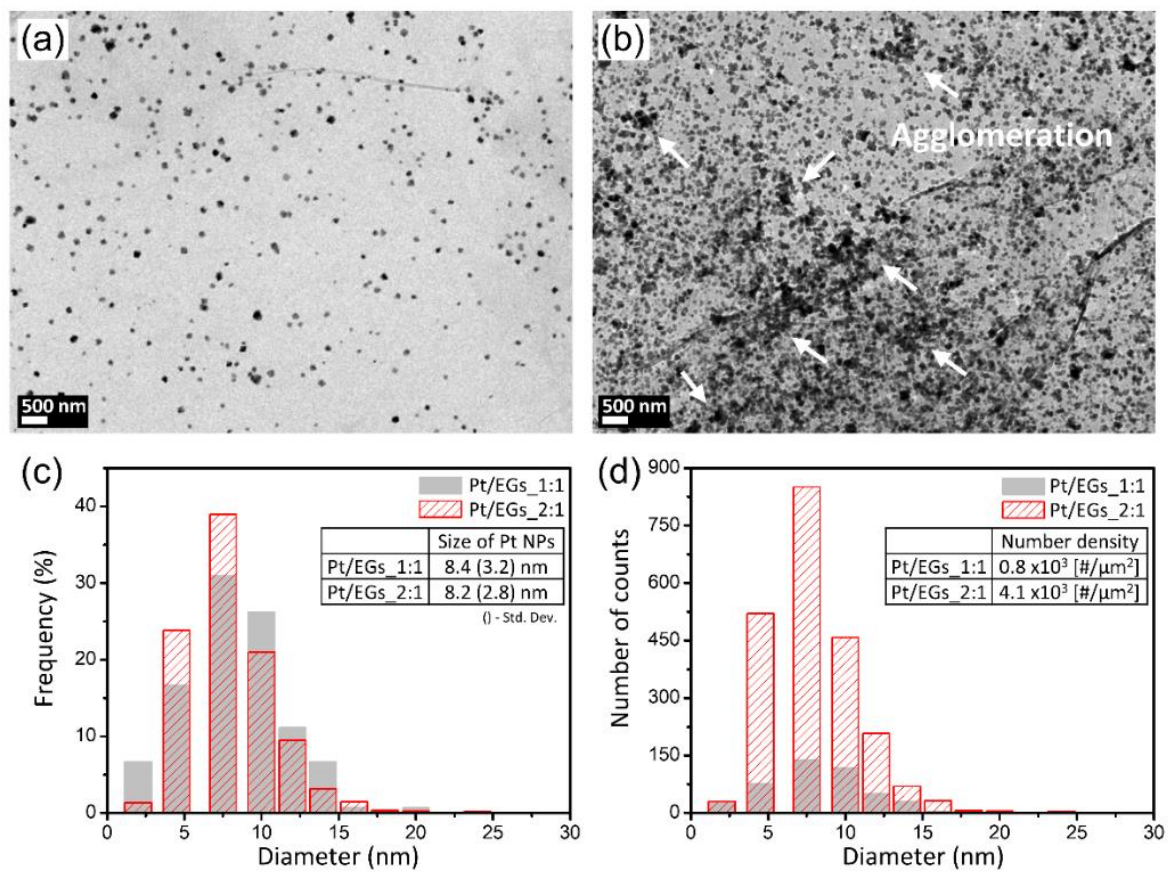

Figure S2. Low magnification HR-TEM images (a) 1:1 and (b) 2:1 weight ratio Pt/EGs catalysts with (c-d) size distributions of the Pt NPs. 

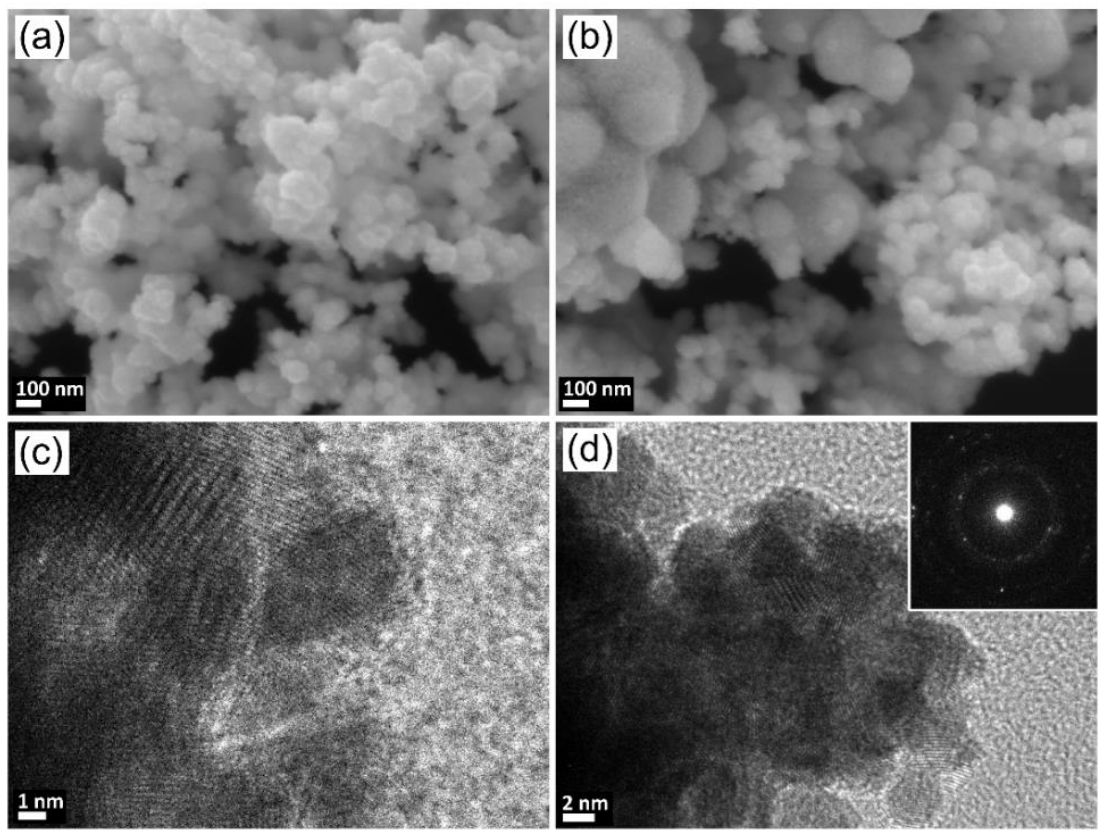

Figure S3. (a-b) FE-SEM and (c-d) HR-TEM images with its FFT pattern of Pt black catalyst.

The Pt black catalyst was composed of Pt nanoparticles with grain sizes of around $3 \mathrm{~nm}$, and it formed hard agglomerates. The secondary particle size was around $50-200 \mathrm{~nm}$. 


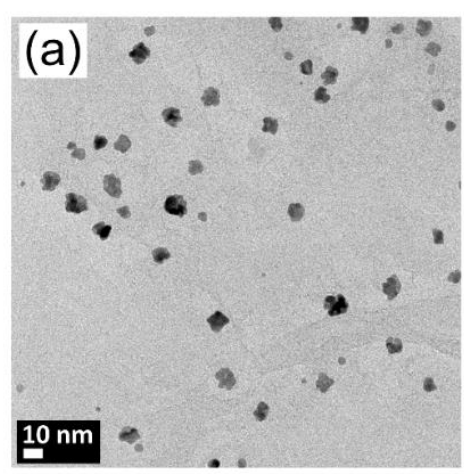

(c)

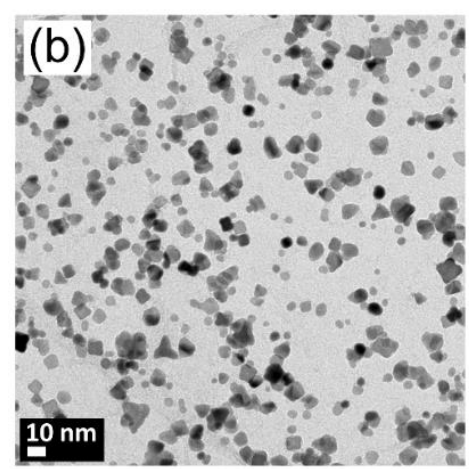

Catalyst layer

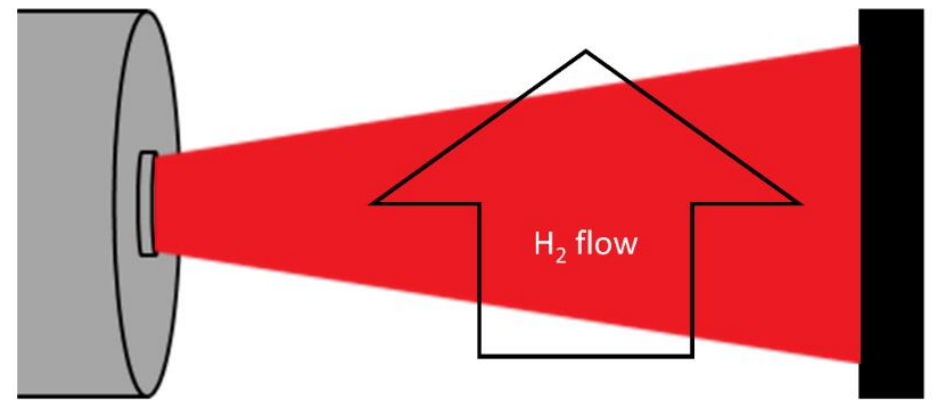

IR-Thermometer

Figure S4. Typical HR-TEM images magnified by $80 \mathrm{k}$ for (a) 1:1 and (b) 2:1 weight ratio Pt/EGs catalysts, and (c) schematic representation of temperature measurement of catalyst during hydrogen oxidation reaction.

The sizes of Pt nano particles (NPs) were measured using the Image $\mathbf{J}$ software by adjusting the contrast of the images. 10 TEM images with a magnification of 80k were prepared for each Pt/EGs sample. Each image was processed using the Image J program. All the information (typically the area of pixels of known distance) was collected, and the mean size was calculated with the size distributions. We assumed that the particles were perfectly round. Since we know the area of exfoliated graphene sheets (EGs) for each image $\left(0.256 \times 0.256 \mu \mathrm{m}^{2}\right)$, we were able to calculate the number density through the number of particles in the images.

The exothermic performance of the Pt/EGs catalysts was measured using an IR-temperature detector and was compared to commercially available Pt black catalyst. To monitor the temperature of the catalysts, the top layer of the cover shown in Fig. S1 was drilled and fitted with an IR-thermometer. The bulk catalyst layers were prepared by dropping $10 \mathrm{mg}$ of each catalyst on a $1 \mathrm{~cm}^{2}$ silicon oxide wafer piece, and $3 \% \mathrm{H}_{2}$ gas in dry air was introduced to the samples. The high loading of Pt NPs on the surface of EGs showed excellent performance over Pt black catalyst. Despite the small primary size of Pt black $(\sim 3 \mathrm{~nm})$, the temperatures of Pt black reached about $70{ }^{\circ} \mathrm{C}$, while the others increased to more than $120^{\circ} \mathrm{C}$. For comparison of the exothermic performance, exothermic response was calculated as $\left(T_{h}-T_{a}\right) / T_{a} * 100$, where $T_{h}$ is saturated temperature in hydrogen atmosphere and $T_{a}$ is saturated temperature in dry air. 

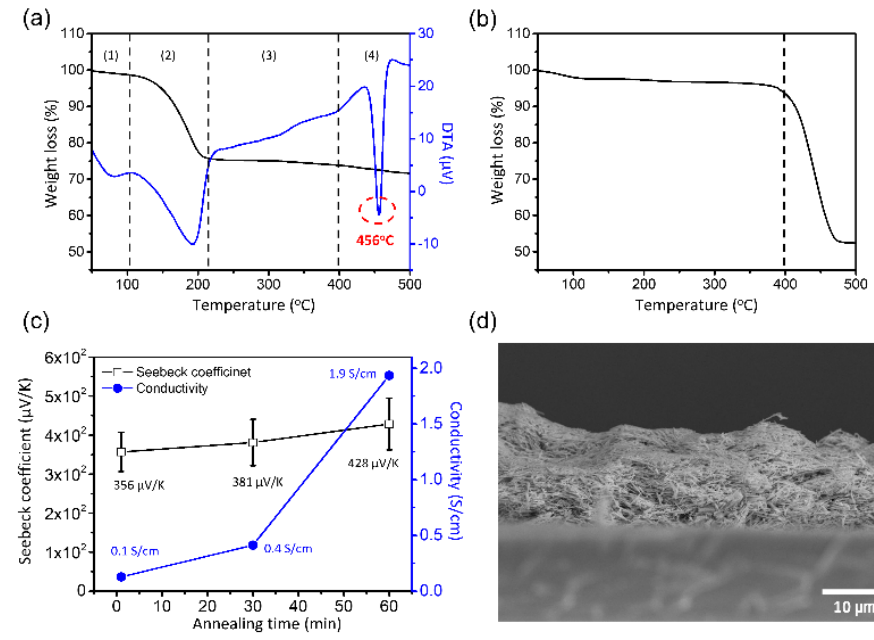

(d)

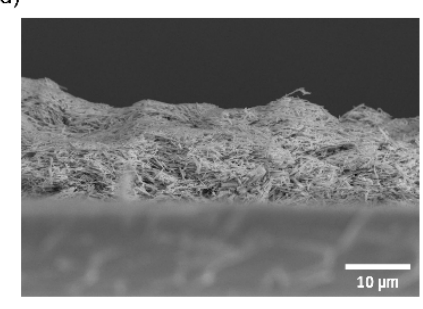

Figure S5. TG-DTA curves for (a) Te paste and (b) PVP measured at $\mathrm{N}_{2}$ atmosphere with a heating rate of $5{ }^{\circ} \mathrm{C}$ per min. Typical FE-SEM images of tellurium based-thermoelectric (TNTE) layer (c) annealed at $300^{\circ} \mathrm{C}$ for $1 \mathrm{~h}$ and (d) $400^{\circ} \mathrm{C}$ for $1 \mathrm{~h}$.

The optimum composition of the paste was determined to be $75 \mathrm{wt} . \%$ Te NWs with a $25 \mathrm{wt} . \%$ solvent mixture composed of 1: 0.8: 0.2 (DEG: DEGBE: PVP). When the Te NW content was less than 75wt.\%, cracks were observed during drying at an ambient temperature for $24 \mathrm{~h}$, whereas when the Te NW content exceeded $75 \mathrm{wt} . \%$, the paste was too viscous to cast on the silicon oxide wafer, and the adhesion was weak.

The characteristics of Te NW-based paste were analyzed by thermogravimetric (TGA) and differential thermal analyses (DTA) and are shown in Figure S5 (a). The thermal behavior of Te paste was divided into four stages, denoted as (1) - (4). In stage (1), slight weight loss was observed and was attributed to the evaporation of adsorbed water molecules. Then in stage (2), evaporation of the solvent mixture should be the main reason for rapid weight loss since the boiling points of DEG and DEGBE are $193{ }^{\circ} \mathrm{C}$ and $171{ }^{\circ} \mathrm{C}$, respectively. In stage (3), there is no distinct change until $300{ }^{\circ} \mathrm{C}$ and when the temperature exceeded $300{ }^{\circ} \mathrm{C}$, slight weight loss was observed. This is regarded as a consequence of evaporation of Te due to its high vapor pressure and relatively low melting point of $449.5^{\circ} \mathrm{C}$. In stage (4), an endothermic reaction peak was clearly observed around $456{ }^{\circ} \mathrm{C}$, which was attributed to the melting of tellurium. Figure S5 (b) confirms that the weight loss in stage (4) is also caused by thermal decomposition of PVP. Therefore, we chose $300{ }^{\circ} \mathrm{C}$ as an optimum annealing temperature because the solvent can evaporate completely at least $193{ }^{\circ} \mathrm{C}$ and relatively high temperature annealing below the melting or evaporation can induce sintering of Te NWs.

In Figure S5 (c), The Seebeck coefficient was increased as annealing temperature increased. This is probably because the Te NWs started to form grain boundaries between wires that as annealing time increased. The increase in conductivity also supports this hypothesis. In Figure S5 (d), it is clearly that Te NWs evaporated when annealed at $400{ }^{\circ} \mathrm{C}$, reducing the thickness down to $\sim 10 \mu \mathrm{m}$. When the annealing time was longer than $60 \mathrm{~m}$ (ex, $90 \mathrm{~m}$ ) or annealing temperature increased to $350^{\circ} \mathrm{C}$, the layer was peeled off from the substrate. 

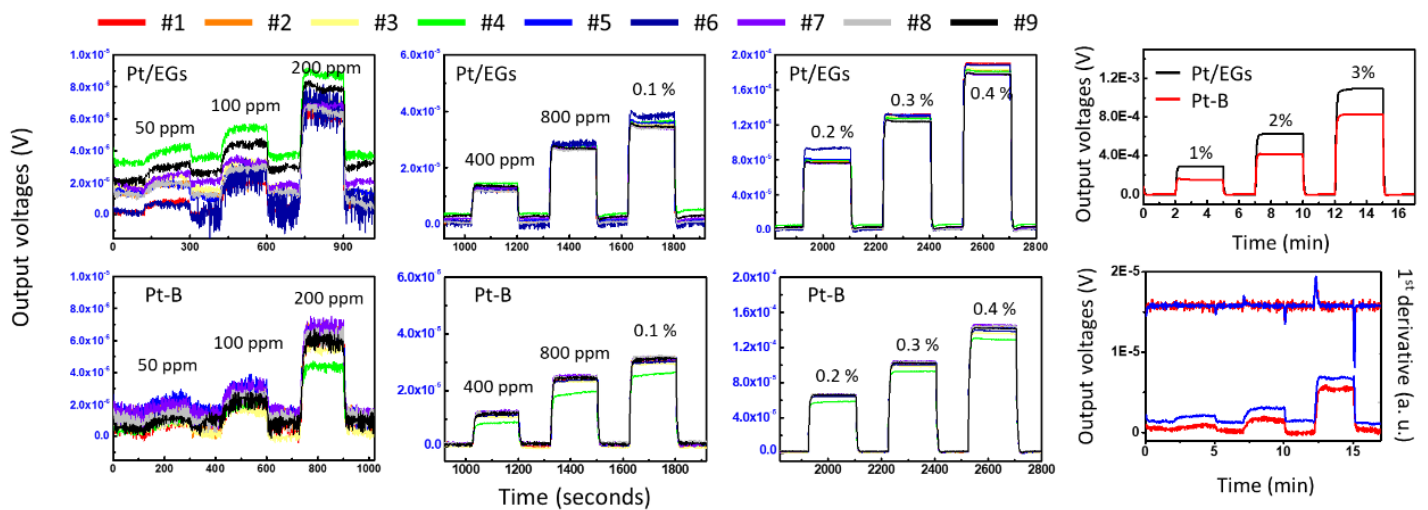

\begin{tabular}{|c|c|c|c|c|c|c|c|c|c|c|}
\hline & ppm & 50 & 100 & 200 & 400 & 800 & 1000 & 2000 & 3000 & 4000 \\
\hline \multirow{4}{*}{ Pt-B } & Response time (s) & - & $26.2(12.0)$ & $16.4(3.8)$ & $14.8(2.9)$ & $12.2(1.4)$ & $10.8(0.9)$ & $9.5(0.8)$ & $9.7(0.6)$ & $9.7(0.6)$ \\
\hline & Recovery time (s) & - & $16.4(18.9)$ & $12.4(2.6)$ & $8.4(1.2)$ & $8.2(1.1)$ & $8.2(0.6)$ & $8.0(0.0)$ & $8.6(0.9)$ & $8.6(0.9)$ \\
\hline & Output voltage $(\mu \mathrm{V})$ & - & $2.40(0.43)$ & $6.02(0.70)$ & $11.5(1.1)$ & $23.8(1.6)$ & $30.4(1.6)$ & $64.5(2.4)$ & $100(3.3)$ & $140(5.0)$ \\
\hline & RSD of output voltage (\%) & - & 18 & 11.6 & 9.9 & 6.7 & 5.3 & 3.8 & 3.3 & 3.6 \\
\hline \multirow{4}{*}{$\mathrm{Pt} / \mathrm{EGs}$} & Response time (s) & 41.9 (23.9) & $28.0(5.5)$ & $13.6(2.5)$ & $10.7(0.9)$ & $8.0(0.9)$ & $7.1(1.0)$ & $5.3(0.9)$ & $5.3(0.9)$ & $6.0(0.0)$ \\
\hline & Recovery time (s) & $6.9(3.0)$ & $6.4(1.3)$ & $4.4(1.6)$ & $4.0(0.0)$ & $4.2(1.5)$ & $3.8(0.6)$ & $4.9(1.0)$ & $4.0(0.0)$ & $5.1(1.0)$ \\
\hline & Output voltage $(\mu \mathrm{V})$ & $2.16(1.06)$ & $3.32(0.96)$ & $6.88(0.82)$ & $12.8(0.82)$ & $27.3(0.77)$ & $35.4(1.5)$ & $80(5.00)$ & $127(2.99)$ & $182(4.6)$ \\
\hline & RSD of output voltage (\%) & 49 & 28.9 & 11.9 & 6.4 & 2.8 & 4.3 & 6.2 & 2.3 & 2.5 \\
\hline
\end{tabular}

Figure S6. All the measured response transient of THS sensors to dry hydrogen gas mixture with calculated mean response time, recovery time, output voltage, and RSD of output voltage values.

Before the test runs, as-fabricated devices were stabilized in dry air for $30 \mathrm{~min}$. To eliminated absorbed water molecules and other impurities at the surface of the catalyst, 3\% of hydrogen gas in dry air was introduced for $5 \mathrm{~min}$ following with flow of dry air for $1 \mathrm{~h}$. Each exposure cycle (50 ppm to $0.4 \% \mathrm{of}_{2}$ gas) was conducted over intervals of $1 \mathrm{~h}$ in dry air. Nine total cycles were done for each device. The stabilization of signals was confirmed by the first derivative trends.

As mentioned in the experimental section, the response and recovery time was calculated as follows. The base line (or base voltages stabilized in dry air) was defined as the mean of output voltage values obtained for $20 \mathrm{~s}$ (typically 11 data) before hydrogen gas was introduced. Likewise, the saturated output voltage value was the mean of the output voltage values obtained for $20 \mathrm{~s}$ (typically 11 data) before the hydrogen gas was stopped. 


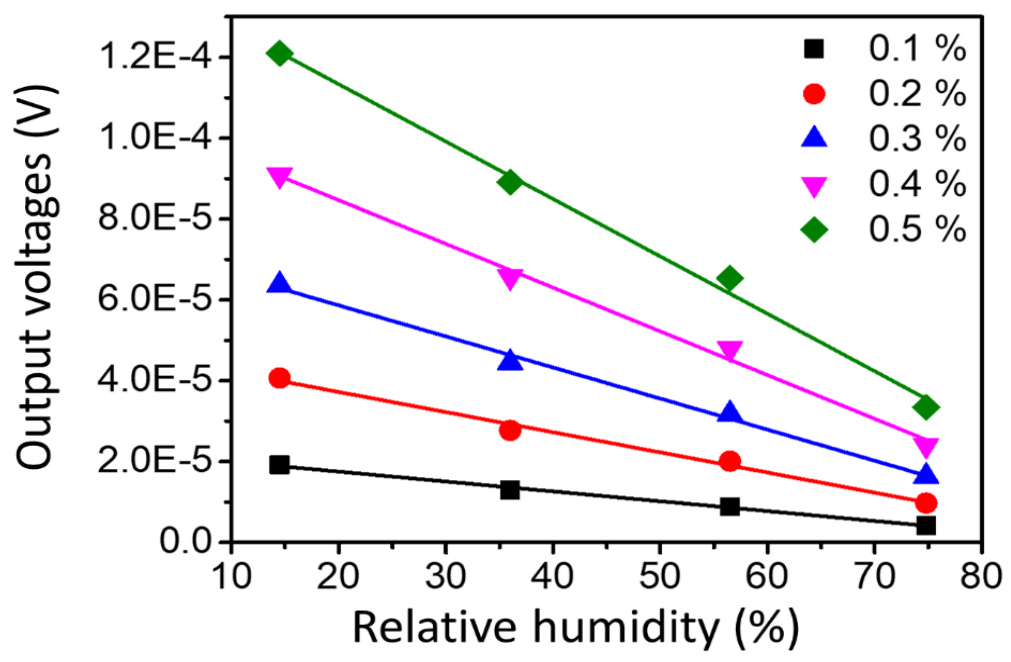

Figure S7. Linear fit data of output voltages as a function of relative humidity. The R-square values were around 0.99 .

(a)

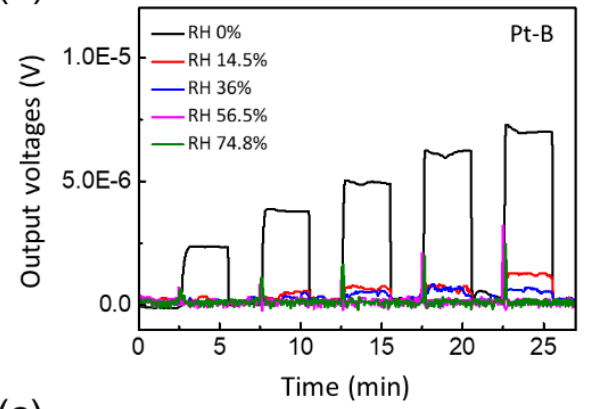

(c)

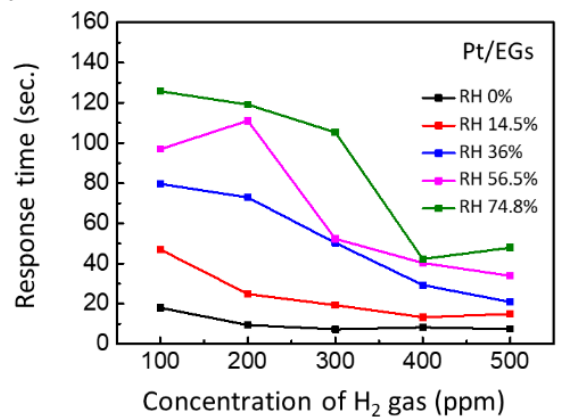

(b)

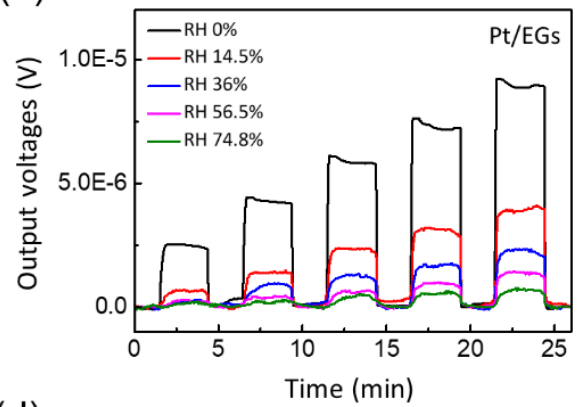

(d)

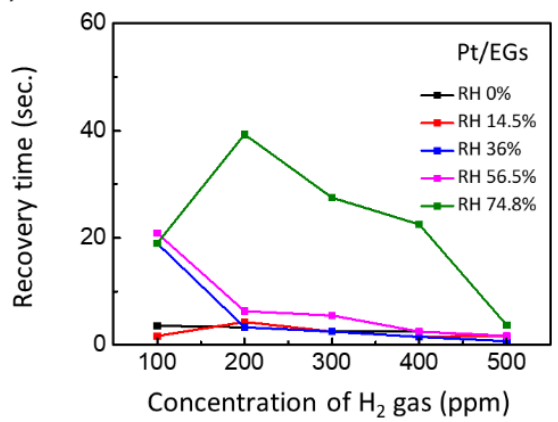

Figure S8. Response transient of (a) Pt-B and (b) Pt/EGs sensors exposed to hydrogen concentrations from 100 to $500 \mathrm{ppm}$ in humid air conditions. (c-d) Calculated response and recovery time of $\mathrm{Pt} / \mathrm{EGs}$ sensor. 

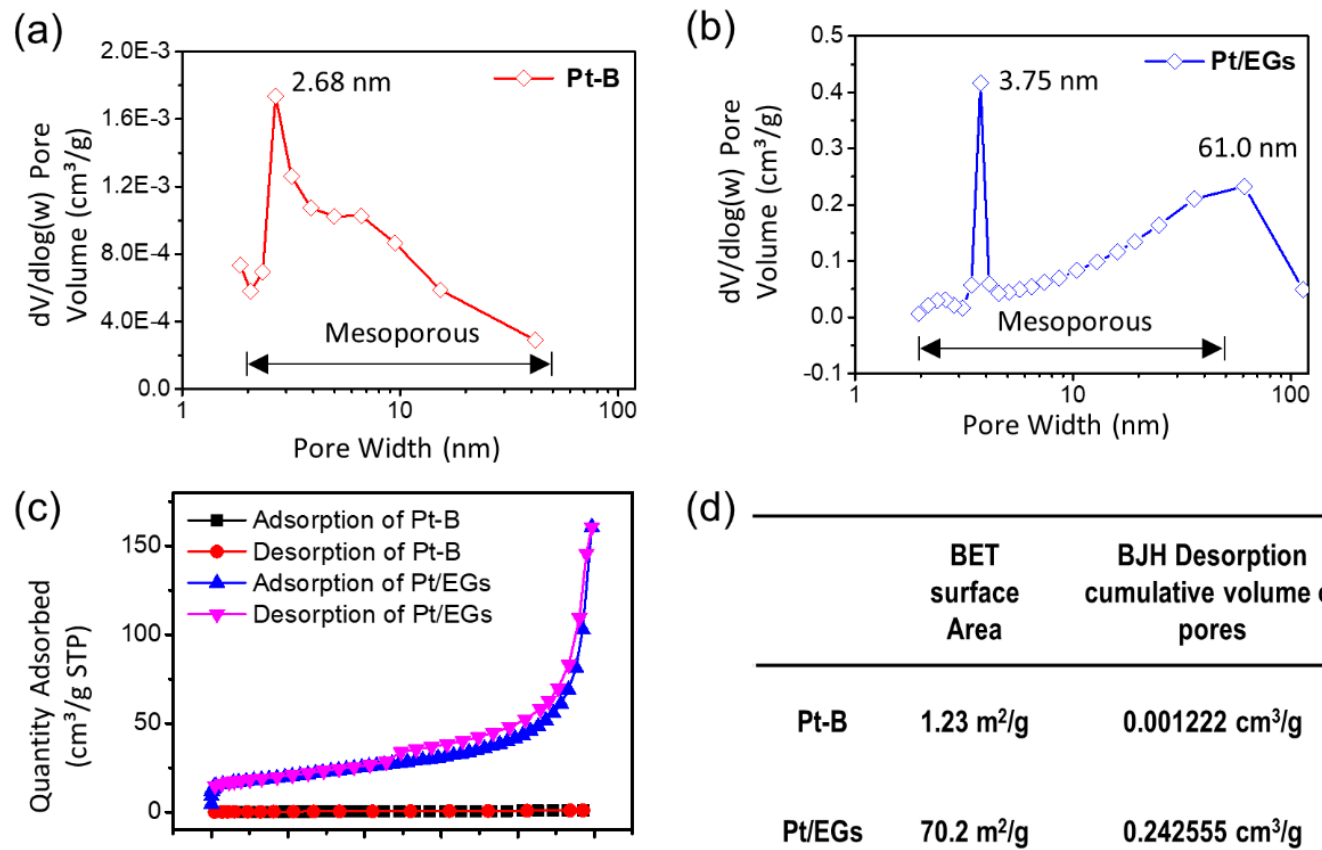

(d)

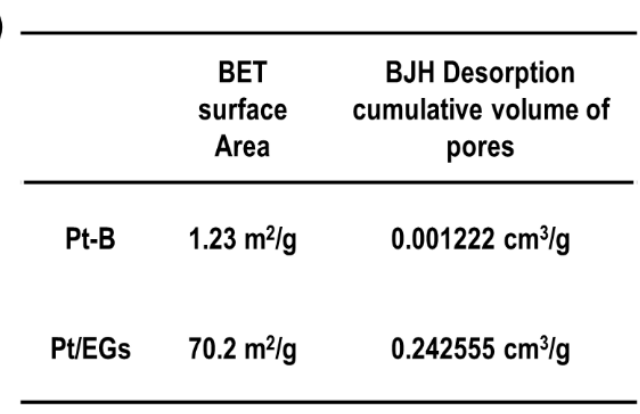

Figure S9. Pore-size distributions of (a) Pt-B and (b) Pt/G catalysts measured by (c) Ar and $\mathrm{N}_{2}$ adsorption/desorption isotherms. (d) Table of corresponding BET surface areas and BJH desorption cumulative volume of pores. 